\title{
Surface critical behavior in Ising magnets: Exact results
}

\author{
D. B. Abraham ${ }^{1}$ and A. Maciołek ${ }^{2}$ \\ ${ }^{1}$ Theoretical Physics, Department of Physics, University of Oxford, 1 Keble Road, Oxford OX1 3NP, United Kingdom \\ ${ }^{2}$ Institute of Physical Chemistry, Polish Academy of Sciences, Department III, Kasprzaka 44/52, PL-01-224 Warsaw, Poland
}

(Received 13 April 2006; published 29 June 2006)

\begin{abstract}
An exact derivation of the surface magnetizations of the two phases $m_{1}^{I}$ and $m_{1}^{I I}$ coexisting below the bulk critical temperature for the semi-infinite two-dimensional Ising ferromagnet subject to a surface field is given. The surface critical behavior of the difference $m_{1}^{I}-m_{1}^{I I}$ is that of the ordinary transition only in a limit of a weak surface field below the wetting temperature.
\end{abstract}

DOI: 10.1103/PhysRevE.73.066129

PACS number(s): 05.50.+q, 68.35.Rh, 64.60.Fr

\section{INTRODUCTION}

Our work is motivated by recent Monte Carlo simulation studies of the surface critical behavior of a model water and a Lennard-Jones fluid in the slitlike and cylindrical pores with a weakly attractive surface $[1,2]$. In the vicinity of surfaces the bulk universality class of critical phenomena splits into several surface universality classes, depending upon whether the tendency to order in the surface is smaller or larger than in the bulk $[3,4]$. In the case of a one-component fluid the presence of a wall should decrease the net fluidfluid attraction between a molecule and its nearest neighbors below the bulk value, which corresponds to a reduced tendency to order at the surface. In a magnetic language this is described by a surface scaling field $c>0$. On the other hand, the wall exerts an effective potential on a fluid which in a magnetic language corresponds to some nonzero surface field $h_{1}$. Thus it is expected that fluids like water or a model Lennard-Jones fluid should lie in the universality class of, so called, "normal" transition, that is labeled by $h_{1}=\infty$ and $c$ $=\infty$. At the normal transition the order parameter (OP) at the surface layer $m_{1}$ should have a leading thermal singularity of the same form as the bulk free energy [5-7]

$$
m_{1}-\left(m_{1 C}+A_{1} \tau+A_{2} \tau^{2}+\cdots\right) \approx+A_{2-\alpha}^{ \pm}||^{2-\alpha},
$$

as $\tau \equiv\left(T_{c}-T\right) / T_{c} \rightarrow 0$, and the contribution in parentheses is a regular background. The authors of Refs. [1,2] focused on the subcritical regime and studied the temperature dependence of the local OP, defined as $\Delta \rho(z) \equiv\left[\rho_{l}(z)-\rho_{v}(z)\right] / 2$, where $\rho_{l}(z)$ and $\rho_{v}(z)$ are the density profiles of the coexisting liquid and vapor phases, respectively, along the pore liquid-vapor coexistence curve. They found that below the bulk critical temperature $T_{c}$ this OP exhibits behavior which is not in accordance with the normal transition, but does exhibit the "ordinary" transition behavior $[3,4]$. This corresponds to vanishing surface field and the reduced tendency to order at the surface and is labeled by $h_{1}=0$ and $c=\infty$, i.e., near the surface a variation of $\Delta \rho$ with reduced temperature $\tau=\left(T-T_{c}\right) / T_{c}$ follows the scaling law with a value of the exponent close to the $\beta_{1} \simeq 0.82$ of the ordinary transition in the Ising system in three dimensions $(d=3)$, i.e.,

$$
\Delta \rho_{1}(\tau) \approx \tau^{\beta_{1}} .
$$

On the basis of these observations the authors put forward the hypothesis that the difference $\Delta \rho$ between the densities of coexisting phases near the surface should follow the behavior (2) for any attractive surfaces below the wetting temperature $T_{w}$. This is based on the assumption that the term $\sim \tau^{\beta_{1}}$ should always be present in both coexisting phases below $T_{w}$. The authors of Refs. [1,2] reconsider the surface critical behavior of the semi-infinite Ising model claiming that below the wetting temperature the surface magnetizations $m_{1}^{I}$ and $m_{1}^{I I}$ in the two phases along the coexistence curve should have the following limiting behavior for $\tau$ small, subcritical:

$$
m_{1}^{I}\left(\tau, h_{1}\right)=B_{1} \tau^{\beta_{1}}+m_{1 C}\left(h_{1}\right)+A_{1}^{\prime}\left(h_{1}\right) \tau+\cdots+A_{2-\alpha}^{-}\left(h_{1}\right)|\tau|^{2-\alpha},
$$

$$
\begin{aligned}
m_{1}^{I I}\left(\tau, h_{1}\right)= & -B_{1} \tau^{\beta_{1}}+m_{1 C}\left(h_{1}\right)+A_{1}^{\prime}\left(h_{1}\right) \tau+\cdots+A_{2-\alpha}^{-}\left(h_{1}\right) \\
& \times|\tau|^{2-\alpha},
\end{aligned}
$$

where $m_{1 C}\left(h_{1}\right)$ is the value of the surface magnetization at the critical point. The symmetric term $\sim \tau^{\beta_{1}}$, which describes the temperature dependence of the magnetization at $h_{1}=0$, accounts for the missing-neighbor effect and, as the authors claim, was overlooked in Refs. [6,7]. Above the wetting temperature there exist a single phase which is expected to have the surface magnetization of the form given by the above equation. The exact results for the boundary magnetization available for the semi-infinite system in the presence of the surface field were derived by McCoy and $\mathrm{Wu}$ and also by Au-Yang and Fisher using a Pffafian method in two dimensions $(d=2)[8,9]$ and concern the behavior only for one of the two possible bulk phases. The temperature dependence of the difference between the magnetizations of both coexisting phases near the surface has not been studied in detail. This is due to the fact that in the absence of the bulk magnetic field the choice of the sign of $h_{1}$ breaks the symmetry in the finite system, and, for example, the positive surface field yields $(+)$ phase in the bulk in the thermodynamic limit. In order to calculate the boundary magnetization for the case of the $(-)$ bulk phase in the presence of the positive boundary field, one would have to perform calculations in the presence of infinitesimally small negative bulk field and put $h \rightarrow 0^{-}$after taking the thermodynamic limit or to solve the model with zero bulk field but with suitable boundary conditions; these boundary conditions are depicted in Fig. 1. In the present paper the latter is done and the surface magnetizations $m_{1}^{I}$ 

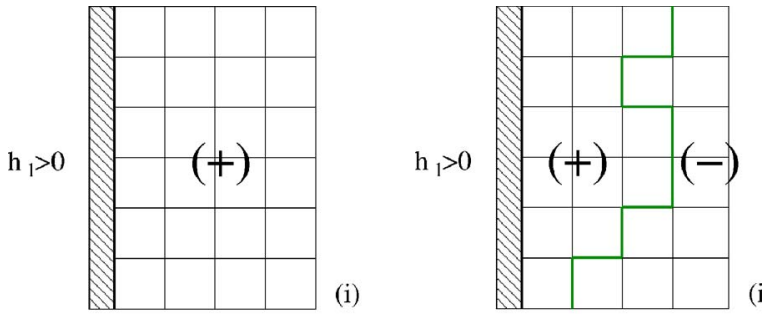

(ii)

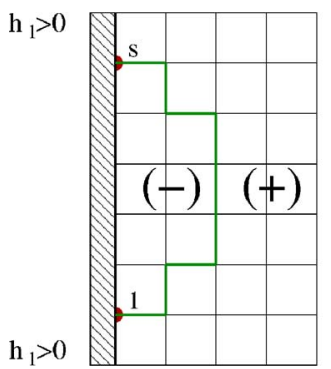

(iii)

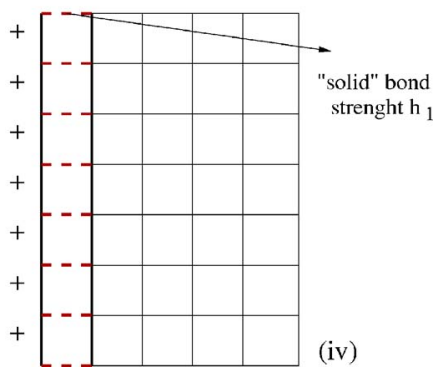

FIG. 1. (Color online) Semi-infinite Ising lattice with boundary conditions to induce (i) surface magnetization $m_{1}^{I}$ and (ii) surface magnetization $m_{1}^{I I}$. (iii) The equivalent version of (ii) adapted for exact calculations. (iv) The positive surface field $h_{1}$ is introduced by modifying bonds to additional wall of spins fixed at the value +1 .

and $m_{1}^{I I}$ of the two phases coexisting below the bulk critical temperature are calculated exactly.

In the case (ii) of the boundary conditions (see Fig. 1), it is useful to think of a domain wall separating the bulk (-) phase from the surface with $h_{1}>0$, which tends to develop positive average magnetization. At the level of individual spins, we have a long Peierls contour and the "gas" of closed loops. Evidently, if $h_{1}<K_{1}$, the long contour will intersect the "solid" bonds to the greatest degree possible on energetic grounds, but there will be a concomitant loss of entropy. It is known that there is a critical $h_{w}\left(K_{1}, K_{2}\right)$ such that for 0 $<h_{1}<h_{w}\left(K_{1}, K_{2}\right)$ the interface is pinned, but for $h_{1}$ $>h_{w}\left(K_{1}, K_{2}\right)$ it fluctuates freely, so that a $(+)$ phase is intercalated at the surface. At the level of the surface magnetization, (i) and (ii) are the same. We emphasize that this is not so for $0<h_{1}<h_{w}\left(K_{1}, K_{2}\right)$. The magnetization at all distances from the surface has been obtained for both (i) and (ii) by exact calculation [10]. Here we want $m_{1}$, which is not usefully represented by the series given in [10], which is of dispersion type over Fermion number (in this case transfer is normal to the edge). But the scaling as $\tau \rightarrow 0$ and $h_{1} \rightarrow 0$ is clear:

$$
s-\lim \left(m^{*}\right)^{-1} m\left[x / \xi_{b}(\tau), h_{1} / \tau^{1 / 2}\right]=f_{q}(\bar{x}, \bar{h}),
$$

where $s$-lim means the coupled limit $h_{1} \rightarrow 0, \tau_{1} \rightarrow 0$ such that $\bar{h}=h_{1} / \tau^{1 / 2}$ is fixed, and $x \rightarrow \infty$ such that $\bar{x}=x / \xi_{b}$ is also fixed. In the above $m^{*}$ is the spontaneous magnetization of the bulk and $q$ indexes whether case (i) or (ii) is under consideration.

The calculation reported in this paper follows case (iii) in Fig. 1. The thermodynamic limit is taken, followed by the limit $s \rightarrow \infty$. This just recaptures a completely spin-reversed version of (ii), but in the controlled way to ensure that correct bulk phase is obtained.

\section{FORMULATION}

Consider a $d=2$ Ising model on a square lattice $(M+1)$ $\times N$ with ferromagnetic couplings (in units of $k_{B} T$ ) $K_{1}$ and $K_{2}$ along bonds in the $(0,1)$ and $(1,0)$ directions, respectively. We shall always set the bulk field to zero and consider two different boundary conditions. In case (a) the surface field (in units of $\left.k_{B} T\right) h_{1}>0$ is applied at the left edge ( $x$ $=1)$ of the lattice whereas all spins at the right edge $(x=M$ +1 ) are fixed at the value +1 so that in the limit of the semi-infinite system there is a pure phase with the spontaneous magnetization $+m^{*}$ in the presence of the positive surface field. In case (b) the field is reversed at the right edge except between points $(1,1)$ and $(1, s)$, whereas all spins at the left edge are fixed at the value -1 so that in the limit of the semi-infinite system and $s \rightarrow \infty$ we have the pure phase with the bulk spontaneous magnetization $-m^{*}$ in the presence of the positive surface field.

If the site $i$ belongs to the left edge of a lattice, the surface magnetization, $m_{1}$, is defined by $m_{1} \equiv\left\langle\sigma_{i}\right\rangle$ (where $\langle\cdot\rangle$ is the ensemble average). To evaluate the spin expectation we use the standard transfer matrix (TM) theory $[11,12]$. Results obtained for the direction of the transfer taken to be parallel to the edge with the applied field are particularly transparent. For case (a) the surface one-point function is

$$
m_{1}^{I}=\lim _{M \rightarrow \infty} \lim _{N \rightarrow \infty} \frac{\operatorname{Tr} \sigma_{1}^{x}\left(V_{2} V_{1}\right)^{N}}{Z^{+}}
$$

where $Z^{+}$is the partition function

$$
Z^{+}=\lim _{N \rightarrow \infty} \operatorname{Tr}\left(V_{2} V_{1}\right)^{N}
$$

$\sigma_{i}^{\alpha}(\alpha=x, y, z)$ is the $\alpha$-component Pauli operator acting on site $i(0 \leq i \leq M+1)$. The transfer matrix $V_{2}$ is

$$
V_{2}=\exp \left(h_{1} \sigma_{0}^{x} \sigma_{1}^{x}+K_{2} \sum_{j=1}^{M} \sigma_{m}^{x} \sigma_{m+1}^{x}\right),
$$

whereas the transfer matrix $V_{1}$ is

$$
V_{1}=\left(2 \sinh 2 K_{1}\right)^{M / 2} \exp \left(-K_{1}^{*} \sum_{j=1}^{M} \sigma_{j}^{z}\right) .
$$

Note that there are no $j=0$ or $j=M+1$ terms in the above sum because the associated edges are hard and have $K_{1}^{*}=0$. Here the dual variable $K_{1}^{*}$ is the real positive solution of $e^{-2 K_{1}^{*}}=\tanh K_{1}$. Both operators $V_{1}$ and $V_{2}$ are self-adjoint. We shall use the following symmetrization of the transfer matrix $V^{\prime}=V_{1}^{1 / 2} V_{2} V_{1}^{1 / 2}$. In terms of $V^{\prime}$ the expression for $m_{1}$ takes the form

$$
m_{1}^{I}=\lim _{M \rightarrow \infty} \lim _{N \rightarrow \infty} \frac{\operatorname{Tr} V_{1}^{1 / 2} \sigma_{1}^{x} V_{1}^{-1 / 2} V^{\prime N}}{\operatorname{Tr} V^{\prime N}} .
$$

Since we are interested in large $N$ asymptotics we will focus on the most significant terms in the spectral decomposition of $V^{\prime N}$ to obtain 


$$
m_{1}^{I}(M)=\left\langle\Phi^{+}\left|V_{1}^{1 / 2} \sigma_{1}^{x} V_{1}^{-1 / 2}\right| \Phi^{+}\right\rangle,
$$

where $\left|\Phi^{+}\right\rangle$is the vacuum corresponding to fixed edge spin states $|++\rangle$. For case (b) the surface magnetization is given by

$$
\begin{aligned}
m_{1}^{I I}= & \lim _{s \rightarrow \infty} \lim _{M, N \rightarrow \infty} \operatorname{Tr} R(0,0)\left(V_{2} V_{1}\right)^{s / 2} \sigma_{1}^{x}\left(V_{2} V_{1}\right)^{s / 2} \\
& \times R(0,0)\left(V_{2} V_{1}\right)^{N-s} / Z^{-},
\end{aligned}
$$

where $Z^{-}$is the partition function for a system with a domain wall between points $(1,1)$ and $(1, s)$ :

$$
Z^{-}=\lim _{s \rightarrow \infty} \lim _{M, N \rightarrow \infty} \operatorname{Tr} R(0,0)\left(V_{2} V_{1}\right)^{s} R(0,0)\left(V_{2} V_{1}\right)^{N-s} .
$$

$R(0,0)$ denotes the spin flip at site $(0,0)$. In terms of $V^{\prime}$ and as $N \rightarrow \infty$ we have

$$
m_{1}^{I I}=\frac{\left\langle\Phi^{+}\left|R(0,0) V^{\prime s / 2} V_{1}^{-1 / 2} \sigma_{1}^{x} V_{1}^{1 / 2} V^{\prime s / 2} R(0,0)\right| \Phi^{+}\right\rangle}{\left\langle\Phi^{+}\left|R(0,0) V^{\prime s} R(0,0)\right| \Phi^{+}\right\rangle} .
$$

Our reason for tackling the problem in this way, with transfer parallel to the edge, is that the boundary conditions will certainly select what will become the bulk $(+)$ phase as $N$ $\rightarrow \infty$. Transfer matrices take convenient forms in terms of spinor operators $\Gamma_{-1}, \Gamma_{0}, \ldots, \Gamma_{2(M+1)}$ defined by $[13]$

$$
\begin{gathered}
\Gamma_{2 j}=\prod_{i=0}^{j-1}\left(-\sigma_{i}^{z}\right) \sigma_{j}^{y}, \quad j=1, \ldots, M+1, \\
\Gamma_{2 j-1}=\prod_{i=0}^{j-1}\left(-\sigma_{i}^{z}\right) \sigma_{j}^{x}, \quad j=1, \ldots, M+1,
\end{gathered}
$$

$\Gamma_{-1}=\sigma_{0}^{x}$, and $\Gamma_{0}=\sigma_{0}^{y}$. The spin flip operator is $R(0,0)=-\sigma_{0}^{z}$ $=i \sigma_{0}^{x} \sigma_{0}^{y}=i \Gamma_{-1} \Gamma_{0}$ and $\sigma_{1}^{x}=i \Gamma_{-1} \Gamma_{0} \Gamma_{1}$. We now use the adjoint action of $V_{i}$ on $\Gamma$,

$$
V_{1}^{1 / 2} \sigma_{1}^{x} V_{1}^{1 / 2}=\left(i \cosh K_{1}^{*} \Gamma_{1}+\sinh K_{1}^{*} \Gamma_{2}\right) \Gamma_{-1} \Gamma_{0},
$$

and expand $\Gamma_{i}$ in Fermi operators $X(k)$ and $X(k)^{\dagger}$ that "diagonalize" the transfer matrix $V^{\prime}$, i.e.,

$$
V^{\prime}=\exp \left(-(1 / 2) \sum_{k} \gamma(k)\left[2 X(k)^{\dagger} X(k)-I\right]\right) .
$$

$k=1, \ldots, M$ are the distinct solutions of the equation

$$
e^{2 i M k}=-e^{i \delta(k)} e^{i\left[\delta^{\prime}(k)-k\right]}
$$

where

$$
\begin{gathered}
e^{i \delta(k)}=e^{i \delta^{\prime}(k)}\left(w_{1} e^{i k}-1\right) /\left(e^{i k}-w_{1}\right), \\
e^{i \delta^{\prime}(k)}=(A B)^{-1 / 2}\left[\frac{\left(e^{i k}-A\right)\left(e^{i k}-B\right)}{\left(e^{i k}-A^{-1}\right)\left(e^{i k}-B^{-1}\right)}\right]^{1 / 2} .
\end{gathered}
$$

In the above $A=\exp 2\left(K_{1}+K_{2}^{*}\right), B=\exp 2\left(K_{1}-K_{2}^{*}\right)$, and $w_{1}$ is the wetting parameter given by

$$
w_{1}=e^{2 K_{1}}\left(\cosh 2 K_{2}-\cosh 2 h_{1}\right) / \sinh 2 K_{2},
$$

where $0<h_{1}<K_{2}$ and $\gamma(k)$ is the Onsager function with $\gamma(k)>0$ for real $k$ :

$$
\cosh \gamma(k)=\cosh 2 K_{1}^{*} \cosh 2 K_{2}-\sinh 2 K_{1}^{*} \sinh 2 K_{2} \cos k .
$$

The expansion gives

$$
\Gamma_{i}=\sum_{k} N_{k}\left[y_{k, i} X^{\dagger}(k)+y_{k, i}^{*} X(k)\right] .
$$

$N_{k}$ are normalization factors which can be determined from the orthogonality of the basis $\left(X_{k}, X_{k}^{\dagger}\right)$. The coefficients $y_{k, i}$ and $y_{k, i}^{*}$ that appear in such a decomposition are

$$
\begin{gathered}
y_{2 j-1}(k)=e^{i \delta(k)} e^{-i(j-1) k}+e^{i \delta^{\prime}(k)} e^{i j k}, \\
i y_{2 j}(k)=e^{i \delta(k)} e^{i \delta^{\prime}(k)} e^{-i(j-1) k}+e^{i j k},
\end{gathered}
$$

for $j=1, \ldots, M$ with the modified results

$$
\begin{gathered}
y_{0}(k)=i \frac{\sinh 2 h_{1} \cosh K_{1}^{*}}{\sinh \gamma(k)} y_{1}(k), \\
y_{2 M+1}(k)=i \frac{\sinh 2 K_{2} \cosh K_{1}^{*}}{\sinh \gamma(k)} y_{2 M}(k)
\end{gathered}
$$

at the boundary.

\section{RESULTS}

The results for $m_{1}^{I}$ and $m_{1}^{I I}$ are

$$
m_{1}^{I}(M)=i \cosh K_{1}^{*} \sum_{k} N_{k}^{2} y_{k, 0}^{*} y_{k, 1}+\sinh K_{1}^{*} \sum_{k} N_{k}^{2} y_{k, 0}^{*} y_{k, 2}
$$

where c.c. denotes the complex conjugate to $y_{k^{\prime}, 1}^{*} y_{k^{\prime}, 0}$. We should now take the limit $M \rightarrow \infty$ followed by $s \rightarrow \infty$ to approach the thermodynamic limit so as to select the (-) magnetized state in Eq. (30). We find out that the result for the difference $m_{1}^{I I}$ $-m_{1}^{I}$ depends crucially on the temperature range.

(i) Above the wetting temperature given by $w_{1}=1$, i.e., for $w_{1}<1$ there are $M$ distinct real solution of the quantization condition (19) between 0 and $\pi$. Taking the limit $M \rightarrow \infty$ of the difference $m_{1}^{I I}-m_{1}^{I}$ gives 


$$
m_{1}^{I I}-m_{1}^{I}=-2 \sinh 2 h_{1} \cosh ^{2} K_{1}^{*}\left(\frac{1}{(4 \pi)^{2}} \oint e^{-\gamma(k) s / 2} \frac{\mathcal{A}(k)}{\sinh ^{2} \gamma(k)} d k \oint e^{-\gamma\left(k^{\prime}\right) s / 2} \frac{\mathcal{A}\left(k^{\prime}\right)}{\sinh \gamma\left(k^{\prime}\right)} d k^{\prime}\right) / \frac{1}{4 \pi} \oint e^{-\gamma(k) s} \frac{\mathcal{A}(k)}{\sinh ^{2} \gamma(k)} d k,
$$

with

$$
\mathcal{A}(k)=1+e^{i \delta(k)} e^{-i \delta^{\prime}(k)} e^{-i k} .
$$

We want to identify the leading order behavior of Eq. (31) in the limit of $s$ large. The integrals can be evaluated by contour techniques with an appropriate path of integration within the $k$ plane. Examination of Eq. (31) reveals that for this problem we have two competing terms in each integral. A zero of $\mathcal{A}(k)$ and a saddle point (a quasiclassical approximation) coming from the exponentiated term. There are also branch cuts of the Onsager function $\gamma(k)$, which is $2 \pi$ periodic, but they do not provide any leading order behavior. The pole is in the upper half plane at $k=+i v_{1}$ such that $e^{v_{1}}=w_{1}$. Because $\gamma\left(i v_{1}\right)<\gamma(0)=2\left(K_{2}-K_{1}^{*}\right)$ the leading order term of each integral in the Eq. (31) is given by the saddle point at $k=0$ :

$$
\begin{aligned}
\oint e^{-\gamma(k) s} f(k) d k & \approx f^{\prime \prime}(0) e^{-s \gamma(0)} \int_{0}^{\infty} e^{-\gamma^{\prime \prime}(0) s k^{2}} k^{2} d k \\
& =\frac{\sqrt{\pi}}{2} \frac{f^{\prime \prime}(0) e^{-\gamma(0) s}}{\left[\gamma^{\prime}(0) s\right]^{3 / 2}} .
\end{aligned}
$$

In conclusion for large $s$

$$
m_{1}^{I I}-m_{1}^{I} \approx s^{-3 / 2} \rightarrow 0 \quad \text { as } s \rightarrow \infty .
$$

(ii) Below the wetting temperature with $w_{1}>1$, provided $M$ is big enough, there is a purely imaginary solution of the quantization condition (19) $k=i v_{0}=i v_{1}+O\left(e^{-2 M v_{1}}\right)$ and $\gamma\left(i v_{1}\right)$ is the smallest of the $\gamma$ 's. In the limit $M \rightarrow 0$ the contribution to the sums in Eqs. (29) and (30) coming from this imaginary wave number dominates the large $s$ behavior of the difference $m_{1}^{I I}-m_{1}^{I}$ giving the following result:

$$
m_{1}^{I I}-m_{1}^{I} \approx-\cosh ^{2} K_{1}^{*} \sinh 2 h_{1} \frac{1-w_{1}^{-2}}{\sinh \gamma\left(i v_{1}\right)} .
$$

What is the leading singular behavior of the above equation as $\tau \rightarrow 0$ ? If $h_{1}$ is not weak the wetting temperature $T_{w}\left(h_{1}\right)$ lies well below the critical temperature and $\tau$ is not small enough to reach the asymptotic behavior. Figure 2 shows the $\log$-log plot of $\Delta m_{1}$ as a function of $\tau$ for three different surface fields $h_{1}=0.01,0.5$, and 0.8 (the same values as used in Ref. [14] where $\Delta m_{1}$ was studied using density-matrix renormalization-group approach for the Ising model in a strip geometry). One can see that only for $h_{1}=0.01$, for which $\tau_{w} \equiv\left[T_{w}\left(h_{1}\right)-T_{c}\right] / T_{c} \simeq-6 \times 10^{-5}, \Delta m_{1} \sim|\tau|^{1 / 2}$ the dominant scaling behavior corresponding to $\beta_{1}=1 / 2$, i.e., that of the ordinary transition. The "source" of the square-root behavior of Eq. (35) in the limit $\tau, h_{1} \rightarrow 0$ is the term $\gamma\left(i v_{1}\right)$. Indeed, assuming for the simplicity $K_{1}=K_{2} \equiv K$ we have

$$
w_{1}=1-h_{1}^{2}\left(e^{4 K_{c}}+1\right)-4 K_{c} \tau\left(1+h_{1}^{2}\right)+O\left(\tau^{2}, h_{1}^{4}\right),
$$

where $K_{c} \equiv J / k T_{c}$ and

$$
\begin{aligned}
\sinh \gamma\left(i v_{1}\right) & =\left\{\left[\cosh 2\left(K-K^{*}\right)+1-\cosh v_{1}\right]^{2}-1\right\}^{1 / 2} \\
& =\left\{2 h_{1}^{2}+8 K_{c} \tau\left[1+h_{1}^{2}\left(e^{4 K_{c}}+1\right)\right]\right\}^{1 / 2}+O\left(\tau^{2}, h^{4}\right) .
\end{aligned}
$$

Thus in a limit $h_{1} \rightarrow 0$,

$$
m_{1}^{I I}-m_{1}^{I} \approx-2 \sqrt{2 K_{c}} \cosh ^{2} K_{c} \sinh 2 h_{1} \mid \tau^{1 / 2},
$$

where $K_{c}=(1 / 2) \ln (1+\sqrt{2})$. The scaling expression for the difference is

$$
\begin{aligned}
m_{1}^{I I}-m_{1}^{I} \approx & -\left(2^{3 / 2} \cosh ^{2} K_{c}\right) h_{1}^{2}\left[\bar{h}\left(e^{4 K_{c}}+1\right)\right. \\
& \left.+4 K_{c} \bar{h}^{-1}\right] / \sqrt{4 K_{c}+\bar{h}^{2}} .
\end{aligned}
$$

\section{CONCLUSIONS}

Our exact calculations show that the limiting behavior of the surface magnetizations given by Eq. (3) is satisfied only for sufficiently weak surface fields below the wetting tem-

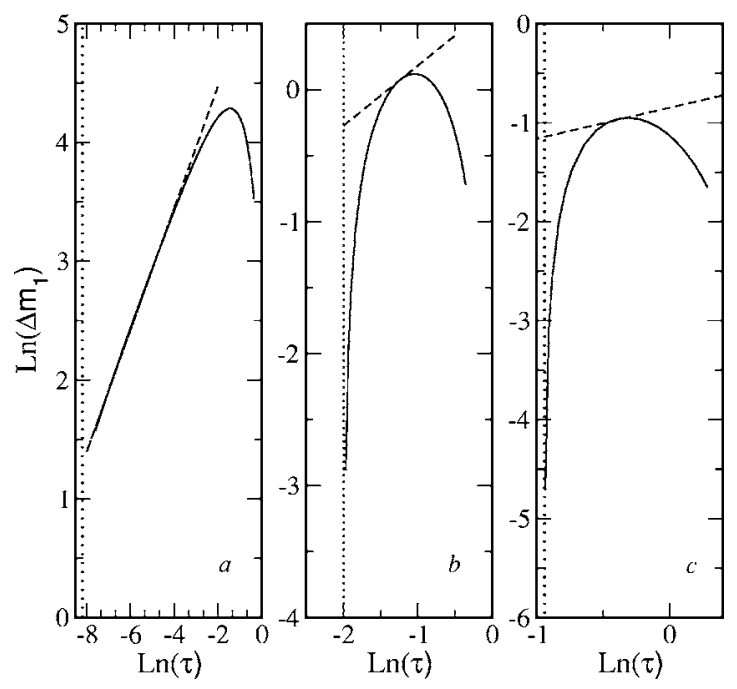

FIG. 2. The log-log plots of $\Delta m_{1}$ as a function of $\tau$ for various surface fields: (a) $h_{1}=0.01$, (b) $h_{1}=0.5$, (c) $h_{1}=0.8$. The vertical dotted lines denote wetting temperatures and the dashed lines present the slope $1 / 2$. 
perature. Above the wetting temperature $\Delta m_{1}=0$ independent of the value of $h_{1}$. Our results fully support the prediction of Refs. [6,7]. The DMRG results reported in Ref. [14] are consistent with our exact results.

\section{ACKNOWLEDGMENT}

This work was partially funded by the EPSRC under Grant No. GR/R83712/01.
[1] I. Brovchenko, A. Geiger, and A. Oleinikova, J. Phys.: Condens. Matter 16, 1 (2004).

[2] I. Brovchenko, A. Geiger, and A. Oleinikova, Eur. Phys. J. B 44, 345 (2005).

[3] For a general review of critical behavior at surfaces, see K. Binder, in Phase Transitions and Critical Phenomena, edited by C. Domb and J. L. Lebowitz (Academic Press, London, 1983), Vol. 8, p. 1.

[4] H. W. Diehl, in Phase Transitions and Critical Phenomena, edited by C. Domb and J. L. Lebowitz (Academic Press, London, 1986), Vol. 10, p. 75, and references therein.

[5] A. J. Bray and M. A. Moore, J. Phys. A 10, 1927 (1977).
[6] T. W. Burkhardt and H. W. Diehl, Phys. Rev. B 50, 3894 (1994).

[7] H. W. Diehl, Ber. Bunsenges. Phys. Chem. 98, 466 (1994).

[8] B. M. McCoy and T. T. Wu, Phys. Rev. 162, 436 (1967).

[9] H. Au-Yang and M. E. Fisher, Phys. Rev. B 21, 3956 (1980).

[10] D. B. Abraham, Phys. Rev. Lett. 44, 1165 (1980).

[11] A. Maciołek and J. Stecki, Phys. Rev. B 54, 1128 (1996).

[12] F. T. Latrémolière, Ph.D. thesis, University of Oxford, UK, 1996 (unpublished).

[13] B. Kaufman, Phys. Rev. 76, 1232 (1949).

[14] A. Drzewiński, A. Maciołek, and K. Szota, J. Phys.: Condens. Matter 18, 5069 (2006). 